O. Engvold (ed.), Transactions of the International Astronomical Union, Vol. XXVB.

(C)2007 IAU. Printed in the United States of America.

\title{
DECEASED MEMBERS
}

The death of 152 members and consultants of the Union have been reported to the Secretariat since the XXIVth General Assembly.

Alik R. Abbasov

Madge G. Adam

David J. Adams

Tord Adolfsson

Kuduvalli Anantharamaiah

A. Noel Argue

Valerij G. Babin

Richard F. Barrow

Orazio Bendinelli

Donald E. Billings

Nikolai S. Blinov

Alfred Bohrmann

Matrona V. Bratijchuk

Pierre Bretagnon

Ezequiel Cabrita

Enrique Chavira

Vladimir E. Chistyakov

Franco Ciatti

Carl Johan Clemedson

A. W.J. Cousins

Raymond A.J. Coutrez

Arthur E. Covington

James Cuffey

Arthur Falnes Davidsen

Merton E. Davies

Hermann Debrunner

Jean Delhaye

Levent Denizman

Vladimir Dermendjiev

John H. deWitt Jr

Muammer Dizer

Peter Dobronravin

Noel A. Doughty

Lawrence Dunkelman

Christian Friedemann

Herbert Friedman

Yuri I. Galperin

Francis F. Gardner

Karl-heinz Gebler

Giuliano Giuricin
Louise F. Jenkins

A. Jensch

Eberhart Jensen

Frank J. Kerr

Evgeni K. Kharadze

Varvara P. Konopleva

Gennadij S. Kosin

Margita Kresakova

Henrik Kristenson

Georgly V. Kuklin

Marius Laffineur

Michael I. Large

Francesco Lucchin

D. C.V. Mallik

Brian F. Marino

Natalia V. Merman

John D. Mihalov

Ivan G. Moiseev

Andrea P. Molchanov

Olexandr Molotaj

Hugo Moreno

Richard F. Mullaly

Nikolai S. Nesterov

Mikhail Nezlin

Peter Nilson

Minoru Oda

John A. O'Keefe

Bernard J. O'Mara

Liisi Oterma

Michiel Daniel Overbeek

Leonid M. Ozernoy

Michael D. Papagiannis

Haim Leib Pekeris

Rosario Peniche

Winfried Petri

Roger H. Peyturaux

John G. Phillips

Chronis Polymilis

Milorad B. Protich

Henry R. Radoski
Kenneth P. Sims

Joseph W. Siry

Alex G. Smith

Pierre B. Souffrin

C. Bruce Stephenson

Robert S. Stobie

Kaj Aa Strand

Mieczyslaw P.R. Subotowicz

Roland Svensson

John W. Swensson

Rozalia Szafraniec

Enrico G. Tanzi

Alexis E. Troche-Boggino

Naum M. Tseytlin

Alexandros Tsioumis

Yutaka Uchida

David J. van Blerkom

Cornelis J. van Houten

Henri van Regemorter

Vladimir Vanysek

Rafik A. Vardanian

A. Vergnano

Natarajan Visvanathan

Sebastian von Hoerner

Gennadij F. Vyalshin

Max Waldmeier

Arthur B.C. Walker Jr

Joseph Weber

Theophile P.F. Weimer

Schame Weniger

W. Wenzel

Kevin C. Westfold

Albert E. Whitford

David T. Wilkinson

Robert Wilson

Paulus Wiyanto

Kenneth O. Wright

Herbert Wroblewski

Arne A. Wyller

Kyung-Loh Yu 
Frederick A. Goldsworthy Eli Gradsztajn J. Mayo Greenberg Jesse L. Greenstein Nikolai P. Groushinsky C. Grubissich Pierre Guerin Kaarle A. Haemeen Anttila John P. Hagen

Robert Hanbury Brown Heinz Herold Miguel Angel Herrera Fred Hoyle Victor A. Hughes Kurt Hunger
Reuven Ramaty

Leo Randic

Grote Reber

John H. Reynolds

Johannes Richter

Daniel Rucinski

John A. Russell

Dror Sadeh

Peter A.G. Scheuer

Philip E. Seiden

Bernard Servan

Branislav M. Sevarlic

P. V. Shcheglov

Inna S. Shcherbina-Samojlova

Rein Silberberg
S. A. Zhevakin Herbert Zook 